\title{
Nanosonosensitizer-Augmented Sonodynamic
} Therapy Combined with Checkpoint Blockade for
Cancer Immunotherapy

This article was published in the following Dove Press journal: International Journal of Nanomedicine

\author{
Xiaoning Lin $\mathbb{D}^{1, *}$ \\ Rong Huang ${ }^{2, *}$ \\ Yanlin Huang ${ }^{1} *$ \\ Kai Wang ${ }^{3}$ \\ Heng $\mathrm{Li}^{1}$ \\ Yiheng Bao' \\ Chaohui $\mathrm{Wu}^{4}$ \\ Yi Zhang ${ }^{5}$ \\ Xinhua Tian' \\ Xiaomin Wang ${ }^{6}$ \\ 'Department of Neurosurgery, Zhongshan \\ Hospital Xiamen University, Xiamen, \\ 361004, People's Republic of China; \\ ${ }^{2}$ Department of Child Health, Women and \\ Children's Hospital, Xiamen University, \\ Xiamen, 36I003, People's Republic of China; \\ ${ }^{3}$ School of Public Health, Xiamen University, \\ Xiamen, 36I I02, People's Republic of China; \\ ${ }^{4}$ Department of Thoracic Surgery, \\ Zhongshan Hospital Xiamen University, \\ Xiamen, 361004, People's Republic of China; \\ ${ }^{5}$ Department of Breast Surgery, Xiamen \\ TCM Hospital, Xiamen, 36I00I, People's \\ Republic of China; ${ }^{6} \mathrm{Fujian}$ Provincial Key \\ Laboratory of Chronic Liver Disease and \\ Hepatocellular Carcinoma, Zhongshan \\ Hospital Xiamen University, Xiamen, \\ 361004, People's Republic of China
}

*These authors contributed equally to this work

Correspondence: Xiaomin Wang Fujian Provincial Key Laboratory of Chronic Liver Disease and Hepatocellular Carcinoma, Xiamen University Affiliated Zhongshan Hospital, Xiamen, 361004, People's Republic of China Emailwxm2203@xmu.edu.cn

Xinhua Tian

Department of Neurosurgery, Zhongshan Hospital Xiamen University, Xiamen, 361004, People's Republic of China Email txhmd@I63.com
Introduction: Sonodynamic therapy (SDT) has good targeting and non-invasive advantages in the treatment of solid cancers, and checkpoint blockade immunotherapy is also a promising treatment to cure cancer. However, their antitumor effects are not sufficient due to some inherent factors. Some studies that combined SDT with immunotherapy or nanoparticles have managed to enhance its efficiency to treat cancers.

Methods: In this work, an effective therapeutic strategy that can potentiate the antitumor efficacy of anti-PD-L1 antibody (aPD-L1) is developed by the use of cascade immunosonodynamic therapy (immuno-SDT). Titanium dioxide $\left(\mathrm{TiO}_{2}\right)$, a nanostructured agent for SDT, sonosensitizer Chlorin e6 (Ce6), and immunological adjuvant $\mathrm{CpG}$ oligonucleotide (CpG ODN), are used to construct a multifunctional nanosonosensitizer $\left(\mathrm{TiO}_{2}-\mathrm{Ce} 6-\mathrm{CpG}\right)$. Then, we conducted in vitro and in vivo experiments to explore the antitumor effect of $\mathrm{TiO}_{2}-$ Ce6-CpG under ultrasound (US) treatment.

Results: The characterization tests showed that the nanosonosensitizers are polycrystalline structure with homogeneous sizes, resulting in a good drug loading efficiency. The innovative nanosonosensitizers $\left(\mathrm{TiO}_{2}-\mathrm{Ce} 6-\mathrm{CpG}\right)$ can not only effectively inhibit tumor growth but also stimulate the immune system to activate the adaptive immune responses, using the $\mathrm{TiO}_{2}$ $\mathrm{Ce} 6$ to augment SDT and the immune adjuvant $\mathrm{CpG}$ to enhance the immune response. After combined with the aPD-L1, the synergistic effect could not only efficiently inhibit the primary tumor growth but also lead to an inhibition of the non-irradiated pre-existing distant tumors by inducing a strong tumor-specific immune response.

Conclusion: In this study, we present an effective strategy for tumor treatment by combining nanosonosensitizer-augmented SDT and aPD-L1 checkpoint blockade. This work provides a promising strategy and offers a new vision for treating malignant tumors.

Keywords: cancer immunotherapy, sonodynamic therapy, titanium dioxide nanoparticles, sonosensitizer, checkpoint blockade

\section{Introduction}

Cancer is still one of the most fatal threats to human health in the world nowadays. ${ }^{1,2}$ Conventional standard cancer treatment protocols, such as surgery, radiotherapy, and chemotherapy, are all unsatisfied to fight against cancer and have several inevitable side effects, including immune system impairment, adverse drug reactions, high cost and patient intolerance. $^{3-7}$ Therefore, exploring other efficient therapeutic modalities with high specificities and low toxicities is urgently expected to eradicate malignant tumors, especially their metastases, and further prevent their recurrence. In recent years, various 
research has been performed on the complicated interactions between cancers and the immune systems, and immunotherapy has shown high potentials in treating some patients with advanced or metastatic tumors. ${ }^{8-10}$ Among the different kinds of cancer immunotherapies, checkpoint blockade immunotherapy using different antibodies, like anti-PD-1 antibody (aPD-1) and anti-PD-L1 antibody (aPD-L1), has become a first-line treatment option for some multiple solid tumors. ${ }^{11-13}$ However, only a small number of patients respond to checkpoint inhibition (the response rates range from $10 \%$ to $40 \%$ for most cancer types) because of its dependence on the pre-existence of tumor-infiltrating $\mathrm{CD} 8^{+}$ $\mathrm{T}$ cells, which limit its broad clinical application. ${ }^{14-17}$ Therefore, strategies that can induce tumor microenvironments to increase $\mathrm{T}$ cell infiltration would be promising ways to sensitize primary or metastatic tumors to checkpoint immunotherapy and increase response rates.

Sonodynamic therapy (SDT), based on ultrasound (US), is a non-invasive therapeutic modality that has been shown to induce antitumor immunity. ${ }^{18-20}$ As one of the emerging therapeutic modalities, unlike some traditional or other promising therapeutic procedures (ie, radiotherapy, ${ }^{4}$ chemotherapy, ${ }^{5}$ photodynamic therapy $(\mathrm{PDT})^{21,22}$ and photothermal therapy (PTT) ${ }^{23,24}$ ), SDT is featured with sufficient tissue-penetrating depth, high therapeutic efficiency, mitigating side effects and low cost, which makes it specific for treating some patients with tumors in deep positions that are challenging to access surgically. ${ }^{25,26}$ The therapeutic mechanism of SDT generally involves the sono-cavitation effect using sonosensitizers and the production of highly toxic reactive oxygen species (ROS), predominantly the singlet oxygen $\left({ }^{1} \mathrm{O}_{2}\right)$, which kills cancer cells directly by inducing necrosis or apoptosis, also known as immunogenic cell death (ICD), and indirectly by damaging vessels or inhibiting neovascularization in tumor tissues and producing tumor-specific immunity. ${ }^{21,27,28}$ Although tumor-associated antigens like peptides or proteins may induce antitumor immune effects under the help of immunologic adjuvants, the existing heterogeneity of patients limits their clinical application. ${ }^{29}$ Besides, previous studies showed that debris from tumor tissues could serve as tumor-associated antigens by using SDT modality to elicit host immune response. ${ }^{18,30}$ However, the immune-oriented efficacy of SDT alone is not yet particularly robust to suppress primary tumor growth and metastasis. ${ }^{30}$ Therefore, developing a new strategy that is sufficiently strong and highly efficient for cancer immunotherapy is necessary.
Herein, we report, on the rational design and construction of a multifunctional nanosonosensitizer $\left(\mathrm{TiO}_{2}-\mathrm{Ce} 6-\mathrm{CpG}\right)$ with the encapsulated sonosensitizers and immunological adjuvant for high efficient cancer immunotherapy, which has been systematically evaluated and demonstrated by both in vitro and in vivo experiments. Titanium dioxide $\left(\mathrm{TiO}_{2}\right)$ is one of the most studied sensitizers in nanostructured material science for PDT and SDT and is non-toxic to live cells due to the advantages of chemical inertness and longterm stability under physiological conditions. ${ }^{31-33}$ Although pure $\mathrm{TiO}_{2}$ nanoparticles $\left(\mathrm{TiO}_{2} \mathrm{NPs}\right)$ are proven to generate ROS under US irradiation effectively, the quantum yield of the ROS production is low. ${ }^{34}$ To improve the ability of $\mathrm{TiO}_{2}$ NPs as a nanosonosensitizer for SDT, it is used as a carrier to load Chlorin e6 (Ce6) and CpG oligonucleotide (CpG ODN) to enhance the immune response. $\mathrm{Ce} 6$ is a hydrophilic sonosensitizer derived from porphyrin, which has been shown to accumulate more effectively in tumors, and could be activated to produce a quantity of ROS to induce apoptosis and necrosis of the tumor cells by the US. ${ }^{35} \mathrm{CpG}$ ODN is an immunological adjuvant that can induce cellular immune responses through the activation of Toll-like receptor 9 (TLR9) and acts as an immunostimulant to enhance the anticancer activity of a variety of cancer treatments. ${ }^{36}$ Upon SDT of primary tumors injected with $\mathrm{TiO}_{2}-\mathrm{Ce} 6-\mathrm{CpG}$, the released tumor-associated antigens could demonstrate vaccine-like functions together with $\mathrm{CpG}$ adjuvant, which can activate dendritic cells (DCs) and increase tumor-infiltrating $\mathrm{CD}^{+} \mathrm{T}$ cells to tumor tissues, generating a robust antitumor immunological response. Inspired by this result, the cascade immuno-SDT was further combined with aPD-L1 checkpoint blockade therapy, which could not only efficiently inhibit the primary tumor growth but also lead to an inhibition of the non-irradiated pre-existing distant tumors via a significant abscopal effect (Figure 1), indicating the potentiated effectiveness of checkpoint blockade antibodies.

\section{Materials and Methods Materials and Reagents}

Titanium dioxide $\left(\mathrm{TiO}_{2}\right)$ nanoparticles and Chlorin e6 (Ce6) were purchased from Sigma-Aldrich. $\mathrm{CpG}$ oligonucleotide (ODN) and FITC-labeled $\mathrm{CpG}$ were purchased from Invivogen. 1,3-diphenylisobenzofuran (DPBF) and 2',7'-dichlorofluorescein diacetate (DCF-DA) were obtained from Sigma-Aldrich. 4',6-diamidino-2-phenylindole was obtained from Beyotime Biotechnology. Anti-PD -L1 (aPd-L1), anti-CD11c and anti-CD86 antibodies were 


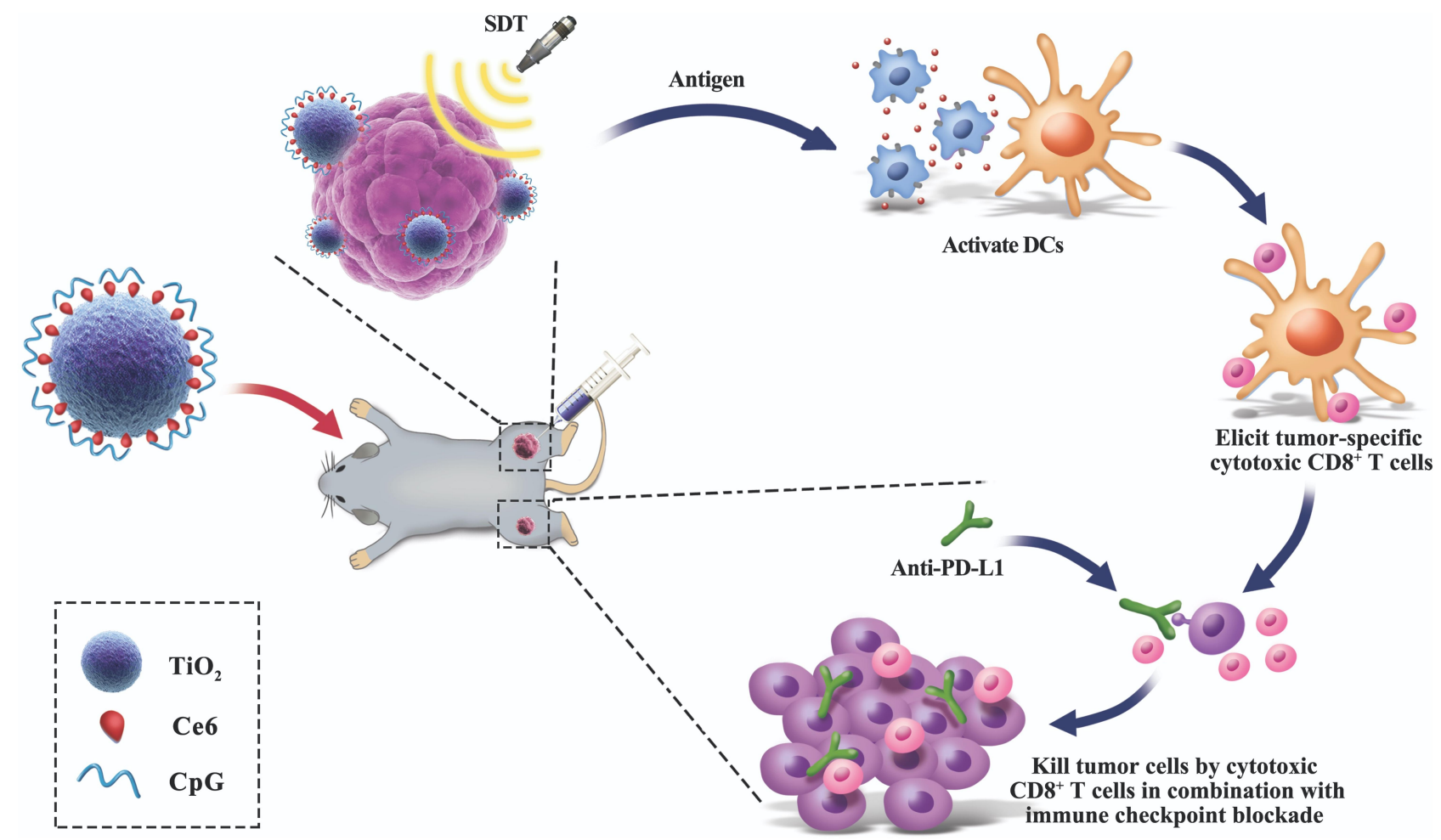

Figure I Schematic illustration of antitumor immunity induced by combined noninvasive SDT with nanosonosensitizers and checkpoint blockade for effective cancer immunotherapy. SDT of nanosonosensitizers $\left(\mathrm{TiO}_{2}-\mathrm{Ce} 6-\mathrm{CpG}_{\mathrm{P}}\right)$ induces ICD at the primary tumor site, leading to the release of tumor-associated antigens. The antigens activate DCs, and then elicit the proliferation of tumor-specific cytotoxic CD8 ${ }^{+} \mathrm{T}$ cells. Combined with PD-LI checkpoint blockade, the SDT of nanosonosensitizers can result in not only tumor eradication in the primary sites but also a systemic antitumor immune response to reject distant tumors.

purchased from Invivogen. Tumor necrosis factor- $\alpha$ (TNF$\alpha$ ) and granulocyte-macrophage colony-stimulating factor (GM-CSF) were obtained from Sigma-Aldrich. For cell culture, RPMI-1640, DMEM, trypsin-EDTA, and fetal bovine serum (FBS) were purchased from Invivogen. All ELISA Kit was purchased from Sigma-Aldrich. All chemicals were of analytical grade and no further purification was required.

\section{Preparation of $\mathrm{TiO}_{2}-\mathrm{Ce} 6-\mathrm{C}_{p} \mathrm{G}$ Nanosonosensitizers}

Briefly, $1 \mathrm{mg} \mathrm{TiO}_{2}$ nanoparticles were dispersed in ultrapure water $(1 \mathrm{~mL})$ to prepare the working stock solution. After sonicated at $50 \mathrm{~W}$ on ice for $2 \mathrm{~min}, 20 \mathrm{ug} / \mathrm{mL} \mathrm{CpG}$ adjuvant or FITC-labeled $\mathrm{CpG}$ adjuvant was added in the $\mathrm{TiO}_{2}$ solution. The mixture was then centrifuged at $1000 \mathrm{rpm}$. After $10 \mathrm{~min}$, the supernatant was removed to collect the precipitate. Then, the ultrapure water and sonosensitizer $\mathrm{Ce} 6$ were added into the precipitate and the final concentration of Ce6 was $200 \mathrm{ug} / \mathrm{mL}$. After sonicated on ice at $30 \mathrm{~W}$ for $2 \mathrm{~min}$ and incubated in a shaker for 2-3 $\mathrm{h}$ at room temperature, the above mixed solution was centrifuged at $1000 \mathrm{rpm}$ for $10 \mathrm{~min}$, and then the supernatant was removed. The newly obtained precipitate was washed twice with ultrapure water and centrifuged again to get $\mathrm{TiO}_{2}-\mathrm{Ce} 6-\mathrm{CpG}$ nanosonosensitizers successfully. Ultimately, $\mathrm{TiO}_{2}-\mathrm{Ce} 6-\mathrm{CpG}$ nanosonosensitizers were suspended in ultrapure water to different concentrations for the following use.

\section{Physicochemical Characterization}

The dynamic particle size and zeta potential of $\mathrm{TiO}_{2}-\mathrm{Ce} 6-$ CpG were measured by dynamic light scattering (DLS) (NanoZS 90, Malvern, USA). The morphology and structure of nanosonosensitizer were observed by transmission electron microscopy (TEM) (Tecnai G2 Spirit BioTwin, FEI, USA). To test the encapsulation efficiency (EE), Ce6 and $\mathrm{CpG}$ in the nanosonosensitizer were respectively detected by UV-vis-near-infrared spectroscopy (UV-vis-NIR, Cary 5000, Agilent, USA) to calculate the EE as the formula: EE (\%)= ((weight of loaded drug)/(weight of initially added drug) $) \times$ 100. Accordingly, the drug loading (DL) was calculated as 
the formula: DL $(\%)=(($ weight of loaded drug $) /($ weight of nanosonosensitizer) $) \times 100$.

\section{Cell Culture}

A murine hepatoma cell line Hepa1-6 was obtained from the Shanghai Institute of Cell Bank (Shanghai, China) and cultured in DMEM containing 10\% FBS, $100 \mathrm{U}$ penicillin and $100 \mathrm{U}$ streptomycin. The cells were cultured in an incubator (Thermo Fisher Scientific) at $37^{\circ} \mathrm{C}$ under a humidified atmosphere containing $5 \% \mathrm{CO}_{2}$.

\section{Cell Viability Assay}

The cytotoxicity of nanosonosensitizers was tested using a colorimetric assay with Cell Counting Kit-8 (CCK-8). 2 $\times 10^{4}$ hepa1-6 cells were respectively seeded in a 96-well flat-bottomed plate and incubated for $32 \mathrm{~h}$. After washing with PBS, the cells were replenished with nanosonosensitizers at various concentrations $(0,20,40,60,100$ and 120 $\mu \mathrm{g} / \mathrm{mL}$ ) and coincubated for $24 \mathrm{~h}$. Then, the medium was withdrawn, and fresh RPMI-1640 medium, together with CCK-8, was added to each well. After incubated for $2 \mathrm{~h}$, the absorbance of each well was evaluated by fluorescence analysis using a microplate reader (Varioskan LUX, Thermo, USA) at a wavelength of $450 \mathrm{~nm}$. Accordingly, the cell viability was calculated by measuring OD values deducting the blank.

\section{Tumor Cellular Uptake of $\mathrm{TiO}_{2}-\mathrm{Ce} 6-\mathrm{C}_{p} \mathrm{G}$ Nanosonosensitizers}

The tumor intracellular endocytosis was observed using confocal laser scanning microscopy (CLSM) (LSM780, Carl Zeiss, Germany). $1 \times 10^{5}$ hepa1-6 cells were respectively seeded in a CLSM-specific culture dishes and incubated for $32 \mathrm{~h}$ at $37^{\circ} \mathrm{C}$. The culture media was then replaced with FITC-labeled $\mathrm{TiO}_{2}$-Ce6-CpG nanosonosensitizers ( $1 \mathrm{~mL}, 30 \mu \mathrm{g} / \mathrm{mL}$ in DMEM) and further incubated for $12 \mathrm{~h}$. Next, the DAPI was added into the culture dishes to stain the cell nuclei for $20 \mathrm{~min}$. Finally, the tumor cells were washed with PBS four times for CLSM observation.

\section{Extracellular ROS Generation}

The extracellular ROS generation was evaluated by chemical oxidation of 1.3-diphenylisobenzofuran (DPBF) using UV-vis-near-infrared spectroscopy (UV-vis-NIR, Cary 5000, Agilent, USA) at different time points. Briefly, DPBF $(2 \mathrm{mg} / \mathrm{mL})$ dissolved in acetonitrile was added into various test sample solutions with an identical concentration of $100 \mu \mathrm{g} / \mathrm{mL}$ in a 96-well plate in triplicate. The mixture was then irradiated by US (US frequency: 1.0 $\mathrm{MHz}$, duty cycle: $50 \%$, power density: $1.0 \mathrm{~W} / \mathrm{cm}^{2}$, time duration: $4 \mathrm{~min}$ ). The control group was prepared with deionized water $(200 \mu \mathrm{L})$ and acetonitrile $(2 \mu \mathrm{L})$.

\section{Intracellular ROS Production}

To detect intracellular ${ }^{1} \mathrm{O}_{2}$ generation, 2',7'dichlorofluorescein diacetate (DCF-DA) was used as a redox fluorescence probe. In brief, hepa1-6 cells were seeded in $35 \mathrm{~mm}$ cell-culture dishes at a density of $4 \times 10^{5}$ cells per dish. After $24 \mathrm{~h}$, the medium containing nanosonosensitizers $(80 \mu \mathrm{g} / \mathrm{mL})$ were added into each dish and incubated for $5 \mathrm{~h}$ at $37^{\circ} \mathrm{C}$. Then, the culture media was replaced by DCF-DA and co-incubated for $30 \mathrm{~min}$. Finally, the cells were washed with PBS three times before exposed to US irradiation (US frequency: $1.0 \mathrm{MHz}$, duty cycle: $50 \%$, power density: $1.0 \mathrm{~W} / \mathrm{cm}^{2}$, time duration: 4 min), and then visualized by CLSM.

\section{In vitro Sonotoxicity Assay}

Hepa1-6 cells were seeded in 96 -well plates $\left(2 \times 10^{4}\right.$ cells per well) in RPMI-1640 medium and incubated for $32 \mathrm{~h}$ to allow the cells to adhere to the plates. Then, nanosonosensitizers with the same concentration of $100 \mu \mathrm{g} / \mathrm{mL}$ were added into each well. After co-incubation with cells for $24 \mathrm{~h}$, the cells were washed with PBS and replenished with RPMI-1640 or DMEM medium. Ultimately, the above-treated cells were irradiated by US (US frequency: $1.0 \mathrm{MHz}$, duty cycle: $50 \%$, power density: $1.0 \mathrm{~W} / \mathrm{cm}^{2}$, time duration: $4 \mathrm{~min}$ ), and then MTT agents were added to the medium in each well, and the cell viability was analyzed by using a microplate reader.

\section{In vitro DC Stimulation Experiment}

Bone marrow-derived DC (BMDC) obtained from 8 -week-old $\mathrm{BALB} / \mathrm{c}$ mice were cultured according to an established method. ${ }^{37}$ In brief, the bone marrow was harvested using RPMI-1640 medium containing 10\% FBS and $50 \mu \mathrm{M}$ 2-mercaptoethanol to flush the femur and tibia. After lysis of the red blood cells, the cells were seeded in $60 \mathrm{~mm}$ bacteria-culture dishes at a density of 1 $\times 10^{6}$ cells per dish. Each dish contained $3 \mathrm{~mL}$ medium with $20 \mathrm{ng} / \mathrm{mL}$ GM-CSF. On day 3 , another $3 \mathrm{~mL}$ medium containing $20 \mathrm{ng} / \mathrm{mL}$ GM-CSF was added into the dishes. Then, half of the culture supernatant was gathered and centrifuged on days 6 and 8 . The cell pellet was redispersed in $3 \mathrm{~mL}$ fresh medium containing $20 \mathrm{ng} / \mathrm{mL}$ GMCSF and added into the original dish again. On day 10, the 
BMDCs were obtained. BMDCs were then incubated with four different groups $\left(\mathrm{TiO}_{2}-\mathrm{Ce} 6-\mathrm{CpG}, \mathrm{TiO}_{2}, \mathrm{TiO}_{2}+\mathrm{Ce} 6\right.$, free $\mathrm{CpG}$ ) containing identical amounts of $\mathrm{Ce} 6$ and $\mathrm{CpG}$. $72 \mathrm{~h}$ later, anti-CD11c and anti-CD86 antibodies were used to stain DCs, and cells were further detected by using flow cytometry (LSRFortessa, BD, USA). The inflammatory cytokines (tumor necrosis factor- $\alpha$ (TNF$\alpha)$ ) in culture supernatant were quantitatively measured for evaluating DC maturation with enzyme-linked immunosorbent assay (ELISA) kits based on vendors' protocols.

\section{In vivo Combined Treatments for Cancer Immunotherapy}

All female C57BL/6 mice (6-8 weeks) were purchased from the Shanghai Laboratory Animal Center of the Chinese Academy of Sciences (SLACCAS) and housed in a suitable environment. All in vivo experiments were performed in accordance with the Guide for the Care and Use of Laboratory Animals (8th edition, International Publication No: 978-0-309-15,400-0) and conducted under the protocols approved by Xiamen University Laboratory Animal Center. Sterilized food and distilled water were available for the mice ad libitum. For the first tumor inoculation, hepa1-6 cells $\left(5 \times 10^{6}\right)$ mixed with matrix gels were subcutaneously injected into the right flank of each female C57BL/6 mouse. For the second tumor inoculation, which was performed 6 days later, hepa1-6 cells $\left(5 \times 10^{6}\right)$ mixed with matrix gels were subcutaneously injected into the left flank of each female C57BL/6 mouse. The tumor volume was calculated according to the following formula: (width ${ }^{2} \times$ length) $/ 2$. Then, these tumor-bearing mice were randomly divided into seven groups $(n=6)$, including: (1) PBS, (2) US, (3) $\mathrm{TiO}_{2}-\mathrm{CpG}+$ aPD-L1 + US, (4) $\mathrm{TiO}_{2}-\mathrm{Ce} 6+$ aPD-L1 + US, (5) $\mathrm{TiO}_{2}-\mathrm{Ce} 6-\mathrm{CpG}+\mathrm{US},(6) \mathrm{TiO}_{2}-\mathrm{Ce} 6-\mathrm{CpG}+$ aPD-L1, (7) $\mathrm{TiO}_{2}-\mathrm{Ce} 6-\mathrm{CpG}+$ aPD-L1 + US. On the 9th day, different formulations were intratumorally (i.t.) injected into the first tumors on the right flank of each mouse. On day 10 , some groups were executed the same parameters of US irradiations (US frequency: $1.0 \mathrm{MHz}$, duty cycle: 50\%, power density: $2.0 \mathrm{~W} / \mathrm{cm}^{2}$; time duration: $7 \mathrm{~min}$ ), and antiPD-L1 antibodies were administered at the dose of $50 \mu \mathrm{g} /$ mouse. To evaluate antitumor effect of the combined treatments, at the end of the study, mice were killed, and tumors were removed, collected and centrifuged for further analysis of mature DCs and $\mathrm{CD}^{+} \mathrm{T}$ cells using flow cytometry. In addition, the blood samples of mice were also collected for analysis of the routine and biochemistry to evaluate the biosafety of nanosonosensitizers.

\section{Statistical Analysis}

All statistical analyses were performed with SPSS 25.0 software. All experiment results were from at least three independent measurements $(n \geq 3)$ and data were presented as the mean \pm standard deviation (SD). Student's $t$-test or Mann-Whitney $U$-test was applied to compare different groups. The level of significance was marked as: $* \mathrm{P}<$ $0.05, * * \mathrm{P}<0.01$.

\section{Results and Discussion Preparation and Characterization of $\mathrm{TiO}_{2}$ -Ce6-CpG}

Hydrophilized $\mathrm{TiO}_{2}$ NPs were used as nanoplatforms and decorated by sonosensitizers (Ce6) and immune adjuvants $\mathrm{CpG}$ ODN based on the ionic bond and electrostatic adsorption. The obtained nanosonosensitizers $\left(\mathrm{TiO}_{2}-\mathrm{Ce} 6-\mathrm{CpG}\right)$ were showed polycrystalline structure with homogeneous sizes according to TEM image (Figure 2A). The average hydrodynamic sizes of $\mathrm{TiO}_{2}$ NPs and nanosonosensitizers were $\sim 65 \pm 41.6 \mathrm{~nm}$ and $\sim 79 \pm 47.7 \mathrm{~nm}$ as measured by DLS, respectively, suggesting that the thickness of the decorated layer was about $14 \mathrm{~nm}$ (Figure 2B). The zeta potentials of $\mathrm{TiO}_{2}$ $\mathrm{NPs}, \mathrm{TiO}_{2}-\mathrm{CpG}$ and nanosonosensitizers were $\sim 15.4 \pm$ $3.1 \mathrm{mV}, \sim 4.5 \pm 0.7 \mathrm{mV}$ and $\sim-12.6 \pm 1.8 \mathrm{mV}$, indicating the successful combination of $\mathrm{Ce} 6$ and $\mathrm{CpG}$ ODN onto the surface of $\mathrm{TiO}_{2} \mathrm{NPs}$ (Figure 2C). The encapsulation rate of $\mathrm{CpG}$ is close to $100 \%$, while the encapsulation rate of $\mathrm{Ce} 6$ is $43.7 \pm 3.8 \%$, and the drug loading of $\mathrm{CpG}$ and $\mathrm{Ce} 6$ is $8.5 \pm 2.3 \%$ and $5.6 \pm$ $1.2 \%$, respectively.

\section{In vitro Cytotoxicity Assessments}

The biocompatibility of different concentrations of nanosonosensitizers was evaluated on hepa1-6 cells by a colorimetric assay using CCK-8. As shown in Figure 2D, cells incubated with various concentrations of $\mathrm{TiO}_{2}$-Ce6$\mathrm{CpG}$ for $24 \mathrm{~h}$ were mostly viable even at the dose of 200 $\mu \mathrm{g} / \mathrm{mL}$, suggesting that nanosonosensitizers were not cytotoxic to cells and possessed excellent biocompatibility. 
A

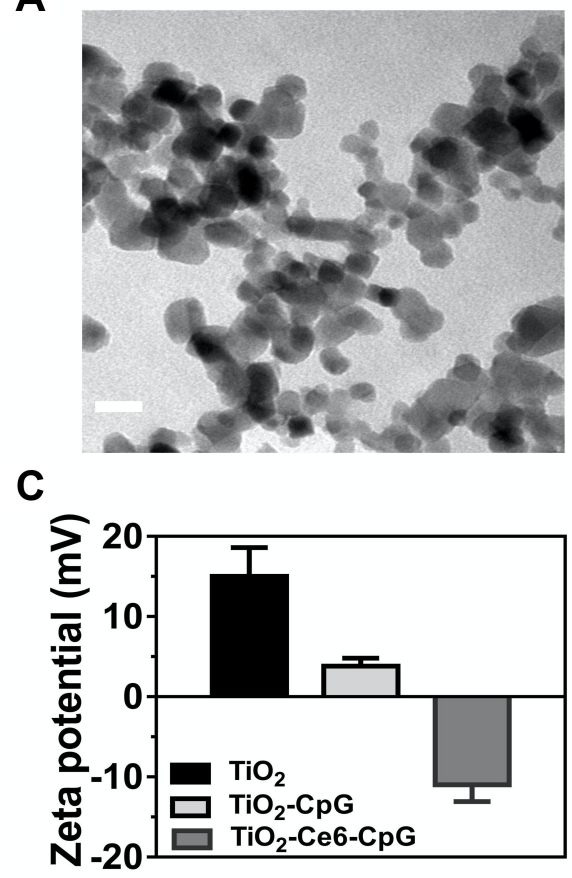

B

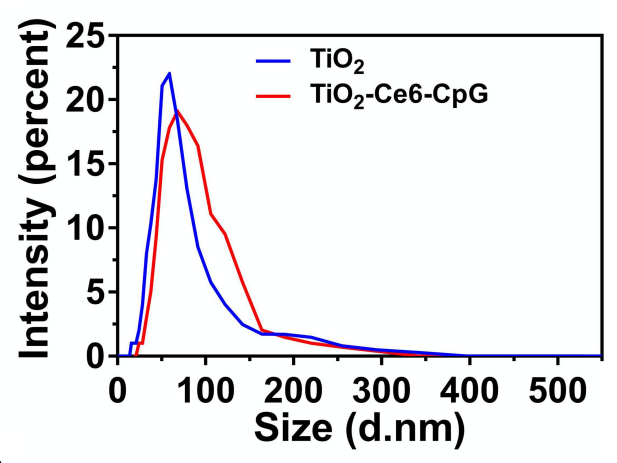

D

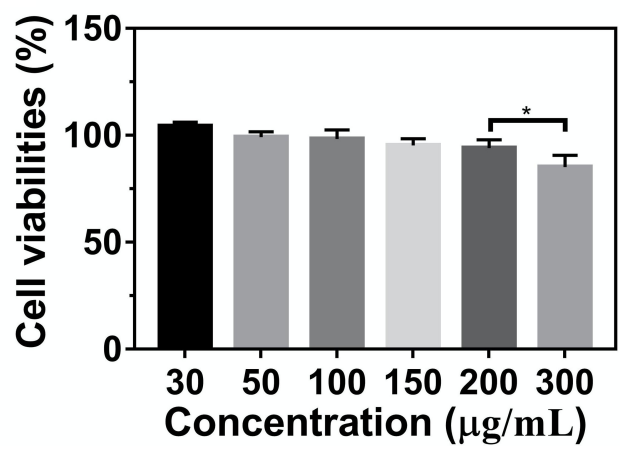

Figure 2 Characterizations and in vitro cytotoxicity of the nanosonosensitizers. (A) TEM image of $\mathrm{TiO}_{2}-\mathrm{Ce}-\mathrm{C}_{\mathrm{PG}}$ nanosonosensitizers $(\mathrm{scale}$ bar $=100$ nm). (B) Hydrodynamic diameters of nanosonosensitisers measured by DLS. (C) Zeta potential of $\mathrm{TiO}_{2}, \mathrm{TiO}_{2}-\mathrm{CpG}_{\mathrm{p}}$ and $\mathrm{TiO}_{2}-\mathrm{Ce}-\mathrm{CpG}$, error bars are based on SD ( $\mathrm{n}=3$ ). (D) Relative viabilities of hepal-6 cells after incubated with different concentrations of $\mathrm{TiO}_{2}-\mathrm{Ce} 6-\mathrm{Cp}_{\mathrm{P}}$ nanosonosensitizers. $* P<0.05$.

\section{Cellular Uptake of Nanosonosensitizers in vitro}

The cellular uptake behaviors of nanosonosensitizers were observed using hepa1-6 liver cancer cells, in which the red, green and blue colors represent $\mathrm{Ce} 6, \mathrm{CpG}$ and DAPI, respectively (Figure 3A). CLSM images showed that $\mathrm{TiO}_{2}$ -Ce6-CpG had an efficient internalization into the cells, implying that nanosonosensitizers could permeabilize the cell membrane.

\section{In vitro ROS Generation}

To detect in vitro ROS generation by nanosonosensitizers under the US irradiation, DPBF degradation assay was employed to quantitatively analyze the production of ${ }^{1} \mathrm{O}_{2}$. As shown in Figure 3B, DPBF had only a small fluorescence drop in the presence of $\mathrm{TiO}_{2}$ NPs or Ce6 alone. However, in the group of $\mathrm{TiO}_{2}-\mathrm{Ce} 6-\mathrm{CpG}$, the amount of fluorescence was demonstrated significant reduction as the US irradiation time increased, indicating that DPBF was consumed quickly by ROS produced by nanosonosensitizers under US irradiation. The results of the efficient ${ }^{1} \mathrm{O}_{2}$ production suggested that
SDT-based therapeutic outcomes would be useful to fight against tumors.

\section{In vitro Antitumor Effect of SDT Using Nanosonosensitizers}

The antitumor effect of $\mathrm{TiO}_{2}-\mathrm{Ce} 6-\mathrm{CpG}$ was assessed by in vitro MTT assay in liver cancer cells (hepa1-6) (Figure 3C). Nanosonosensitizers showed a higher anticancer effect than $\mathrm{TiO}_{2}$ NPs or free Ce6 in tumor cells, which might be due to synergistic enhancement effects of the sonosensitizers $\mathrm{TiO}_{2}$ NPs and Ce6. Moreover, as the time of US irradiation prolonged, stronger cytotoxicity was observed. Therefore, compared to other groups with the same sample concentrations, the combination of nanosonosensitizers and US had a significantly better efficacy to kill tumor cells.

\section{Intracellular ROS Measurement}

To identify whether the antitumor effect of $\mathrm{TiO}_{2}-\mathrm{Ce} 6-\mathrm{CpG}$ under US irradiation originated from the production of ROS, we applied a high-sensitive fluorescent probe (DCF-DA) to 
A
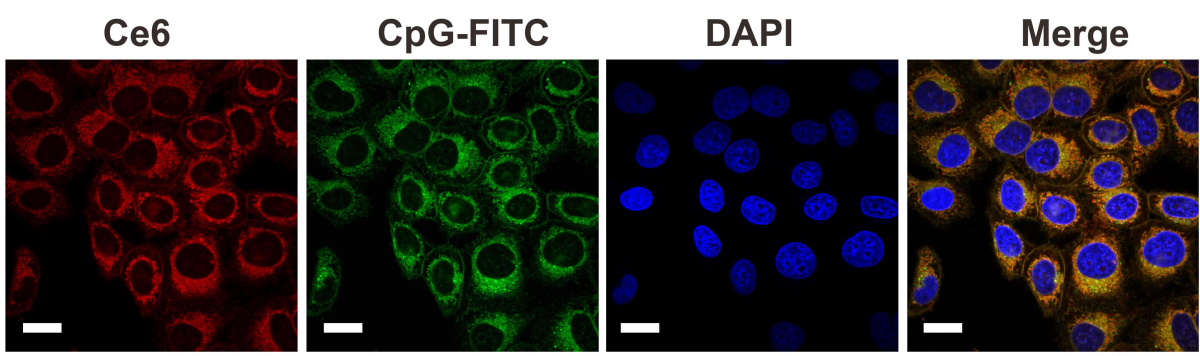

B

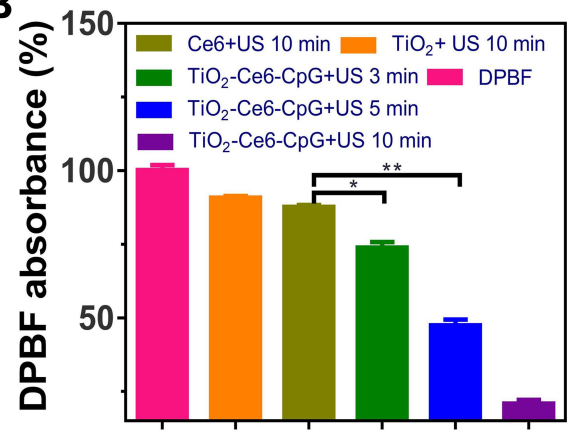

D

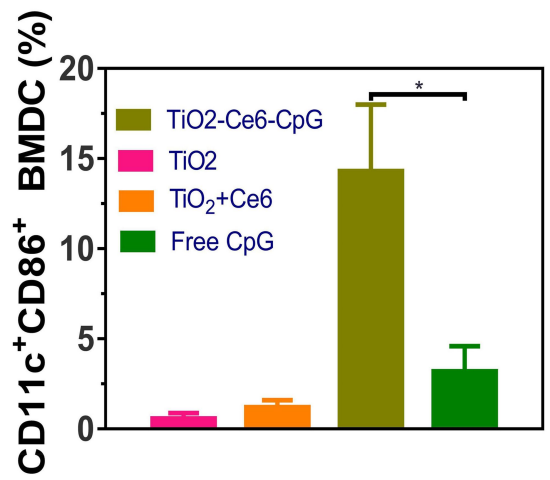

C

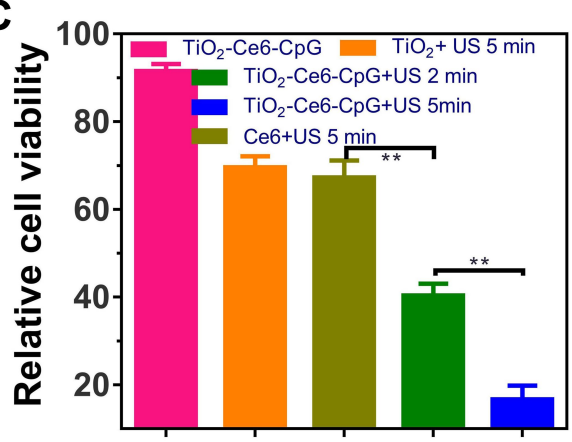

E

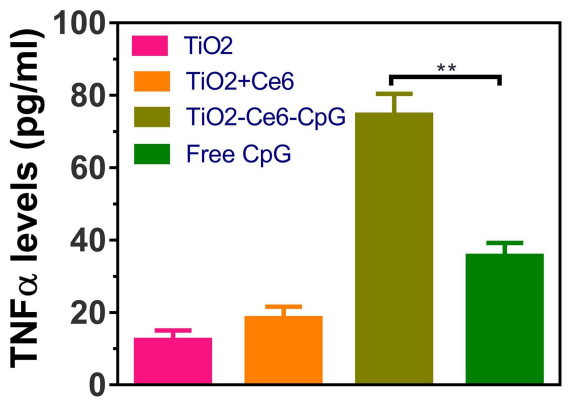

Figure 3 In vitro cellular uptake, ROS generation, antitumor effect and immune response of $\mathrm{TiO}_{2}-\mathrm{Ce}_{-}-\mathrm{Cp}_{\mathrm{pG}}$ nanosonosensitizers. (A) CLSM images of hepal-6 cells after incubation with nanosonosensitizers (scale bar $=15 \mu \mathrm{m}$ ). Nuclei were stained with DAPI. (B) DPBF absorption of $\mathrm{TiO}_{2}, \mathrm{Ce}$ and $\mathrm{TiO}_{2}-\mathrm{Ce} 6-\mathrm{CpG}_{\mathrm{p}}$ under US irradiation. $(\mathbf{C})$ Relative viability of hepa I-6 cells after different treatments, detected by MTT assay. (D-E) Quantification of the level of DC maturation (D) and the secretion of TNF- $\alpha$ (E) in DC suspensions. Data are expressed as means \pm SD $(n=3)$. $* P<0.05$, ** $P<0.01$.

reveal its mechanism (Supplementary Figure 1 and Figure 2). DCF-DA was nonfluorescent and it could be oxidized to fluorescent DCF by intracellular ROS. These results indicated that nanosonosensitizers were able to generate intracellular ROS under the US irradiation to cause cytotoxic effects and further induce the death of tumor cells.

\section{In vitro DC Activation}

To further investigate the function of the component of $\mathrm{CpG}$ ODN in nanosonosensitizers as an immune adjuvant to induce immune response after SDT, flow cytometry was performed to analyze the maturation of DCs. DCs are one of the most important types of antigen-presenting cells and play crucial roles in the process of innate and adaptive immunities. ${ }^{38}$ Once exposure to the antigens, the immature DCs will engulf the antigens and then process them into peptides when migrating to the near lymph nodes. Afterward, the immature DCs will become maturation and present the major histocompatibility complex (MHC) peptide to the naive T cell. ${ }^{39}$ Therefore, the immunological effects of $\mathrm{TiO}_{2}-\mathrm{Ce} 6-\mathrm{CpG}$ towards BMDCs were assessed by analyzing the upregulations of co-stimulatory molecules CD11c and CD86, which are the representative markers for DC maturation. It was found that $\mathrm{TiO}_{2}-\mathrm{Ce} 6-\mathrm{CpG}$ nanosonosensitizers could significantly promote in vitro DC maturation compared with free $\mathrm{CpG}$ ODN at the same dose. Simultaneously, there was no apparent immune-stimulation effect on DCs in the group of $\mathrm{TiO}_{2}$ 
NPs or $\mathrm{TiO}_{2}-\mathrm{Ce} 6$ (Figure 3D). This result also indicated the presence of $\mathrm{CpG}$ ODN in $\mathrm{TiO}_{2}-\mathrm{Ce} 6-\mathrm{CpG}$ nanosonosensitizers.

Cytokine secretion is another important indication of immune responses. ${ }^{40}$ Thus, TNF- $\alpha$, a crucial marker in the activation of cellular immunity, was employed to analyze the level of DCs by ELISA. ${ }^{24,41}$ Consistent with the aforementioned DC maturation results, a significantly higher level of TNF- $\alpha$ was observed by using $\mathrm{TiO}_{2}-\mathrm{Ce} 6-\mathrm{CpG}$ nanosonosensitizers (Figure 3E), further demonstrated that $\mathrm{CpG}$ ODN could enhance the immune response. Therefore, this novel nanosonosensitizer could be used as the immune-stimulating adjuvant and efficiently activated the DC maturation.

\section{In vivo Antitumor Effect of Combined SDT and Immunotherapy}

To study the synergistic therapeutic effect of the combination of nanosonosensitizers-augmented SDT and PD-L1 blockade on primary and metastatic tumors, a bilaterally bearing subcutaneous hepa1-6 tumor model was applied. The design of our experimental animal process is shown in Figure 4A. The liver cancer cells were inoculated on the right flank of each mouse as the primary tumor. Six days later, the second tumor was inoculated on the same mouse's left flank as an artificial model of metastasis. Before the therapeutic experiment, $\mathrm{TiO}_{2}-\mathrm{Ce} 6-\mathrm{CpG}$ nanosonosensitizers were injected into the first tumors on day 9. The following day, the area of the first tumors was treated with US irradiation twice at $24 \mathrm{~h}$ and $48 \mathrm{~h}$. Afterward, mice were intravenously (i.v.) injected with aPD-L1 at doses of $50 \mu \mathrm{g}$ per mouse twice a week for a total of two weeks. The growth of bilateral tumors in different groups was measured by a caliper every other day, and the treatment results are summarized in Figure 4B and C. Notably, $\mathrm{TiO}_{2}-\mathrm{Ce} 6-\mathrm{CpG}$ nanosonosensitizers-augmented SDT, in combination with PD-L1 blockade, could not only efficiently suppress the primary tumor but also significantly inhibit the growth of the distant tumor. To further evaluate the survival outcomes of combined treatment of nanosonosensitizers-augmented SDT and aPD-L1, the tumor-bearing mice were closely monitored after various treatments. We found mice could survive greatly longer for more than 80 days after SDT plus aPD-L1 therapy, in significant contrast to mice in the other six control groups, all of which died within 35-55 days (Figure 4D). These encouraging results suggest that such combined therapy of SDT and non-specific immunotherapy could improve cancer treatment effectively.

In order to show the biosafety of this combined treatment, the evaluation of the potential harmful effect to
A

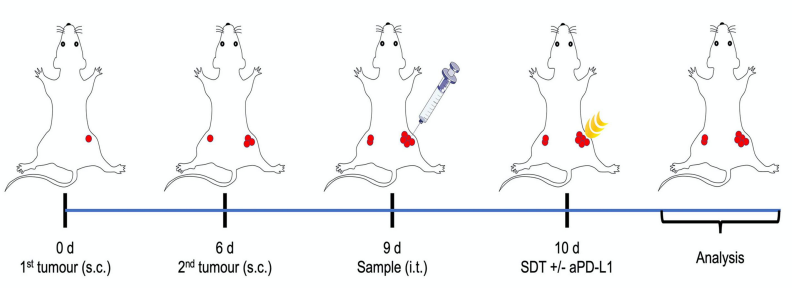

C

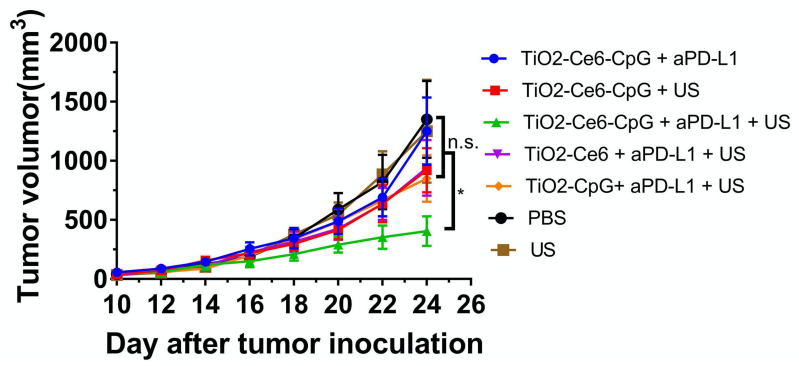

D
B

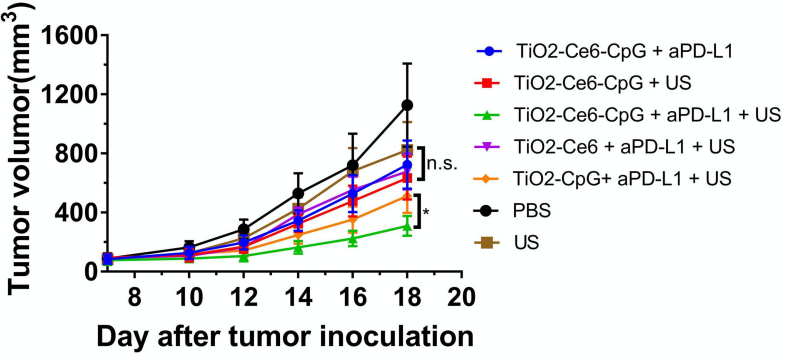

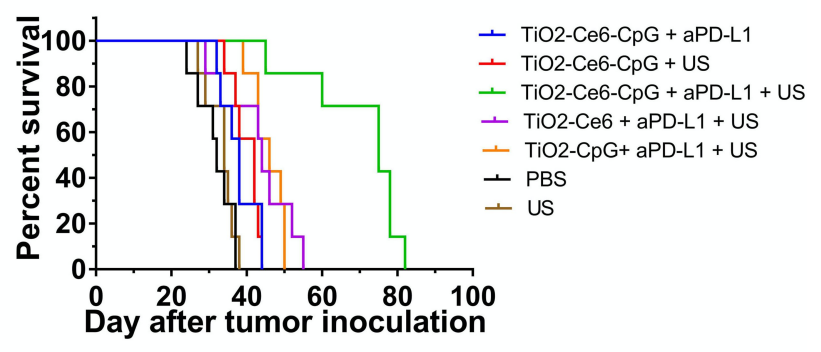

Figure 4 Antitumor effect of nanosonosensitizers-augmented SDT plus PD-LI blockade immunotherapy in subcutaneous tumor models. (A) Schematic illustration of TiO ${ }_{2}-$ Ce6-CpG-based SDT and aPD-LI combination therapy to inhibit tumor growth at distant sites. (B-C) Primary (B) and distant (C) tumor growth curves of different groups of tumor-bearing mice $(n=6)$ after various treatments as indicated in the figure. (D) Morbidity-free survival of different groups of mice-bearing subcutaneous hepal-6 tumors after the indicated treatments $(n=7)$, statistical significance was calculated via the Log rank test. Data are presented as means \pm SD. $* P<0.05$. 

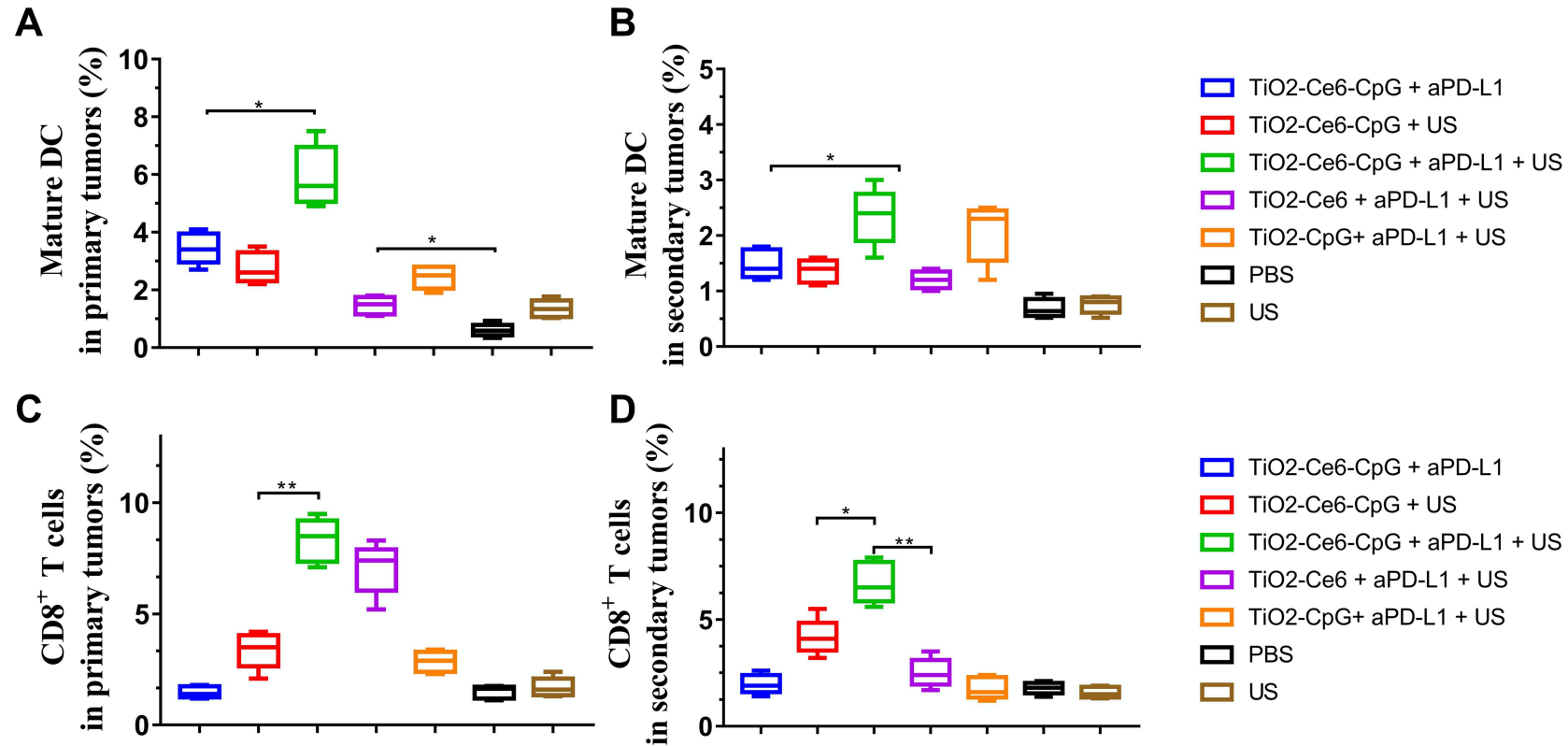

Figure 5 Antitumor immunity after different treatments. The proportion of mature DC in the primary (A) and secondary (B) tumors. Statistical analysis of the proportion of cytotoxic $\mathrm{CD}^{+} \mathrm{T}$ cells in the primary $(\mathbf{C})$ and secondary $(\mathbf{D})$ tumors. Data are expressed as means $\pm \mathrm{SD}(\mathrm{n}=3)$. $* P<0.05, * * P<0.01$.

normal organs was performed. The serum biochemistry and blood routine assays were conducted on the tumorbearing mice after receiving combined treatments. It was observed that there were no significant differences of all the measured indicators between the treated groups and the healthy control (Supplementary Fig. 3-5), indicating that the mice could tolerate such a boosted antitumor immunity of nanosonosensitizers-augmented SDT plus aPD-L1.

Furthermore, to better understand the underlying mechanism behind the antitumor effect, we investigated the abundance of the immune cells (including mature DCs and $\mathrm{CD}^{+} \mathrm{T}$ cells) in the primary and secondary tumor tissues posttreatment. As is well known, DC maturation is critical for antigen presentation to $\mathrm{T}$ cells, thus promoting the infiltration of cytotoxic $\mathrm{CD}^{+} \mathrm{T}$ cells in tumor. We first examined the population of mature DC in both primary and distant tumors after various treatments by flow cytometry. As is shown in Figure 5A and $\mathrm{B}$, treatment with $\mathrm{TiO}_{2}-\mathrm{Ce} 6-\mathrm{CpG}$ $+\mathrm{US}+$ aPD-L1 could significantly enhance the amount of mature DC in bilateral tumors, whereas the phenomenon was not found in the groups of $\mathrm{TiO}_{2}-\mathrm{Ce} 6-\mathrm{CpG}+\mathrm{US}$ or $\mathrm{TiO}_{2}-\mathrm{Ce} 6$ $-\mathrm{CpG}+\mathrm{aPD}-\mathrm{L} 1$. Then, the intratumoral infiltration of cytotoxic $\mathrm{CD}^{+} \mathrm{T}$ cells in both primary and secondary tumors was further analyzed and the similar results were observed (Figure 5C, D and Supplementary Figure 6). The treatment with $\mathrm{TiO}_{2}-\mathrm{Ce} 6-\mathrm{CpG}+\mathrm{US}+$ aPD-L1 also dramatically increased the quantity of $\mathrm{CD}^{+} \mathrm{T}$ cells in both tumors.
These interesting findings verified that combined treatment could not only have synergistic therapeutic effect but also potentiate the infiltration of tumor-specific cytotoxic $\mathrm{CD}^{+}$ T cells, thereby eliciting systematic anti-cancer immunity to kill the distant tumor cells, as is illustrated in Figure 1.

\section{Conclusion}

In summary, we have successfully developed an effective strategy for tumor treatment by combining nanosonosensitizer-augmented SDT and aPD-L1 checkpoint blockade. The innovative nanosonosensitizers $\left(\mathrm{TiO}_{2}-\mathrm{Ce} 6-\mathrm{CpG}\right)$ can not only effectively inhibit tumor growth but also stimulate the immune system to activate the adaptive immune responses, using the $\mathrm{TiO}_{2}-\mathrm{Ce} 6$ to augment SDT and the immune adjuvant $\mathrm{CpG}$ to enhance the immune response. When combined with blockade of PD-L1, SDT of this nanosonosensitizer showed superior inhibitory activity against primary and metastatic tumors in the bilateral subcutaneous mouse model of liver cancer. These delightful results would be attributed to the generation of systematic anti-cancer immunity, including the activated maturation of DCs and effective stimulation of cytotoxic $\mathrm{CD}^{+} \mathrm{T}$ cells. Collectively, this promising strategy offers a new vision for treating malignant tumors.

\section{Acknowledgments}

This research was financially supported by the Medical and Health Key project of Xiamen (grant number: 
3502Z20191106), Joint research project of Science and Technology Bureau and Health Commission of Xiamen (grant number: 3502Z20179046), Joint Fund Science and Technology Department and Health Commission of Fujian Province (grant number: 2019J01562), and President Fund of Xiamen University (grant number: 20720190138).

\section{Disclosure}

The authors report no conflicts of interest in this work.

\section{References}

1. Bray F, Ferlay J, Soerjomataram I, Siegel RL, Torre LA, Jemal A. Global cancer statistics 2018: GLOBOCAN estimates of incidence and mortality worldwide for 36 cancers in 185 countries. CA Cancer J Clin. 2018;68(6):394-424. doi:10.3322/caac.21492

2. Galluzzi L, Kepp O, Vander Heiden MG, Kroemer G. Metabolic targets for cancer therapy. Nat Rev Drug Discov. 2013;12 (11):829-846.

3. Qi J, Chen C, Zhang X, et al. Light-driven transformable optical agent with adaptive functions for boosting cancer surgery outcomes. Nat Commun. 2018;9(1):1848. doi:10.1038/s41467-018-04222-8

4. Ni K, Lan G, Chan C, et al. Nanoscale metal-organic frameworks enhance radiotherapy to potentiate checkpoint blockade immunotherapy. Nat Commun. 2018;9(1):2351. doi:10.1038/s41467018-04703-w

5. Zhang D, Cui P, Dai Z, et al. Tumor microenvironment responsive $\mathrm{FePt} / \mathrm{MoS} 2$ nanocomposites with chemotherapy and photothermal therapy for enhancing cancer immunotherapy. Nanoscale. 2019;11 (42):19912-19922. doi:10.1039/C9NR05684J

6. Li X, Kim J, Yoon J, Chen X. Cancer-associated, stimuli-driven, turn on theranostics for multimodality imaging and therapy. Adv Mater. 2017;29:23.

7. Lin X, Liu S, Zhang X, et al. An ultrasound activated vesicle of janus au-mno nanoparticles for promoted tumor penetration and sono-chemodynamic therapy of orthotopic liver cancer. Angew Chem Int Ed Engl. 2019.

8. Eisenstein M. Immunotherapy offers a promising bet against brain cancer. Nature. 2018;561(7724):S42-S44. doi:10.1038/d41586-01806705-6

9. Matsiko A. Cancer immunotherapy making headway. Nat Mater. 2018;17(6):472. doi:10.1038/s41563-018-0091-8

10. Turajlic S, Larkin J. Immunotherapy for melanoma metastatic to the brain. $N$ Engl $J$ Med. 2018;379(8):789-790. doi:10.1056/ NEJMe1807752

11. Ribas A, Wolchok JD. Cancer immunotherapy using checkpoint blockade. Science. 2018;359(6382):1350-1355. doi:10.1126/science. aar4060

12. Forde PM, Chaft JE, Smith KN, et al. Neoadjuvant PD-1 blockade in resectable lung cancer. $N$ Engl J Med. 2018;378(21):1976-1986. doi:10.1056/NEJMoa1716078

13. Pardoll DM. The blockade of immune checkpoints in cancer immunotherapy. Nat Rev Cancer. 2012;12(4):252-264. doi:10.1038/ nrc3239

14. Ding L, Chen F. Predicting tumor response to PD-1 blockade. N Engl J Med. 2019;381(5):477-479. doi:10.1056/NEJMcibr1906340

15. Auslander N, Zhang G, Lee JS, et al. Robust prediction of response to immune checkpoint blockade therapy in metastatic melanoma. Nat Med. 2018;24(10):1545-1549. doi:10.1038/s41591-018-0157-9

16. Ludin A, Zon LI. Cancer immunotherapy: the dark side of PD-1 receptor inhibition. Nature. 2017;552(7683):41-42. doi:10.1038/ nature 24759
17. Sharma P, Hu-Lieskovan S, Wargo JA, Ribas A. Primary, adaptive, and acquired resistance to cancer immunotherapy. Cell. 2017;168 (4):707-723. doi:10.1016/j.cell.2017.01.017

18. Yue W, Chen L, Yu L, et al. Checkpoint blockade and nanosonosensitizer-augmented noninvasive sonodynamic therapy combination reduces tumour growth and metastases in mice. Nat Commun. 2019;10(1):2025. doi:10.1038/s41467-019-09760-3

19. Zhu P, Chen Y, Shi J. Nanoenzyme-augmented cancer sonodynamic therapy by catalytic tumor oxygenation. ACS Nano. 2018;12 (4):3780-3795. doi:10.1021/acsnano.8b00999

20. Ma A, Chen H, Cui Y, et al. Metalloporphyrin complex-based nanosonosensitizers for deep-tissue tumor theranostics by noninvasive sonodynamic therapy. Small. 2019;15(5):e1804028. doi:10.1002/ smll.201804028

21. Hu L, Cao Z, Ma L, et al. The potentiated checkpoint blockade immunotherapy by ROS-responsive nanocarrier-mediated cascade chemo-photodynamic therapy. Biomaterials. 2019;223:119469. doi:10.1016/j.biomaterials.2019.119469

22. Pei Q, Hu X, Zheng X, et al. Light-activatable red blood cell membrane-camouflaged dimeric prodrug nanoparticles for synergistic photodynamic/chemotherapy. ACS Nano. 2018;12(2):1630-1641. doi:10.1021/acsnano.7b08219

23. Peng J, Xiao Y, Li W, et al. Photosensitizer micelles together with IDO inhibitor enhance cancer photothermal therapy and immunotherapy. Adv Sci (Weinh). 2018;5(5):1700891. doi:10.1002/advs.201700891

24. Chen Q, Xu L, Liang C, Wang C, Peng R, Liu Z. Photothermal therapy with immune-adjuvant nanoparticles together with checkpoint blockade for effective cancer immunotherapy. Nat Commun. 2016;7:13193. doi:10.1038/ncomms13193

25. Canavese G, Ancona A, Racca L, et al. Nanoparticle-assisted ultrasound: a special focus on sonodynamic therapy against cancer. Chem Eng J. 2018;340:155-172. doi:10.1016/j.cej.2018.01.060

26. Rengeng L, Qianyu Z, Yuehong L, Zhongzhong P, Libo L. Sonodynamic therapy, a treatment developing from photodynamic therapy. Photodiagnosis Photodyn Ther. 2017;19:159-166. doi:10.1016/j.pdpdt.2017.06.003

27. Lin X, Song J, Chen X, Yang H. Ultrasound activated sensitizers and applications. Angew Chem Int Ed Engl. 2019.

28. Duan X, Chan C, Lin W. Nanoparticle-mediated immunogenic cell death enables and potentiates cancer immunotherapy. Angew Chem Int Ed Engl. 2019;58(3):670-680. doi:10.1002/anie.201804882

29. Yang J, Zhang Q, Li K, Yin H, Zheng JN. Composite peptide-based vaccines for cancer immunotherapy (Review). Int $J$ Mol Med. 2015;35(1):17-23. doi:10.3892/ijmm.2014.2000

30. Zhang Q, Bao C, Cai X, et al. Sonodynamic therapy-assisted immunotherapy: a novel modality for cancer treatment. Cancer Sci. 2018;109(5):1330-1345. doi:10.1111/cas.13578

31. You DG, Deepagan VG, Um W, et al. ROS-generating TiO2 nanoparticles for non-invasive sonodynamic therapy of cancer. Sci Rep. 2016;6(1):23200. doi:10.1038/srep23200

32. Kim S, Im S, Park EY, et al. Drug-loaded titanium dioxide nanoparticle coated with tumor targeting polymer as a sonodynamic chemotherapeutic agent for anti-cancer therapy. Nanomedicine. 2019;24:102110. doi:10.1016/j.nano.2019.102110

33. Kotagiri N, Sudlow GP, Akers WJ, Achilefu S. Breaking the depth dependency of phototherapy with Cerenkov radiation and low-radiance-responsive nanophotosensitizers. Nat Nanotechnol. 2015;10(4):370-379. doi:10.1038/nnano.2015.17

34. Deepagan VG, You DG, Um W, et al. Long-circulating Au-TiO2 nanocomposite as a sonosensitizer for ROS-mediated eradication of cancer. Nano Lett. 2016;16(10):6257-6264. doi:10.1021/acs.nanolett.6b02547

35. Sun L, Li Q, Hou M, et al. Light-activatable Chlorin e6 (Ce6)-imbedded erythrocyte membrane vesicles camouflaged Prussian blue nanoparticles for synergistic photothermal and photodynamic therapies of cancer. Biomater Sci. 2018;6(11):2881-2895. doi:10.1039/C8BM00812D 
36. Wang C, Sun W, Wright G, Wang AZ, Gu Z. Inflammation-triggered cancer immunotherapy by programmed delivery of $\mathrm{CpG}$ and anti-PD1 antibody. Adv Mater. 2016;28(40):8912-8920. doi:10.1002/adma.201506312

37. Lutz MB, Kukutsch N, Ogilvie AL, et al. An advanced culture method for generating large quantities of highly pure dendritic cells from mouse bone marrow. J Immunol Methods. 1999;223(1):77-92. doi:10.1016/S0022-1759(98)00204-X

38. Janeway CA Jr, Bottomly K. Signals and signs for lymphocyte responses. Cell. 1994;76(2):275-285. doi:10.1016/0092-8674(94) 90335-2
39. Wakim LM, Bevan MJ. Cross-dressed dendritic cells drive memory CD8+ T-cell activation after viral infection. Nature. 2011;471 (7340):629-632. doi:10.1038/nature09863

40. Steinman RM. Decisions about dendritic cells: past, present, and future. Annu Rev Immunol. 2012;30:1-22. doi:10.1146/annurevimmunol-100311-102839

41. He C, Duan X, Guo N, et al. Core-shell nanoscale coordination polymers combine chemotherapy and photodynamic therapy to potentiate checkpoint blockade cancer immunotherapy. Nat Commun. 2016;7:12499. doi:10.1038/ncomms12499

\section{Publish your work in this journal}

The International Journal of Nanomedicine is an international, peerreviewed journal focusing on the application of nanotechnology in diagnostics, therapeutics, and drug delivery systems throughout the biomedical field. This journal is indexed on PubMed Central, MedLine, CAS, SciSearch ${ }^{\mathbb{R}}$, Current Contents ${ }^{\mathbb{R}} /$ Clinical Medicine,
Journal Citation Reports/Science Edition, EMBase, Scopus and the Elsevier Bibliographic databases. The manuscript management system is completely online and includes a very quick and fair peer-review system, which is all easy to use. Visit http://www.dovepress.com/ testimonials.php to read real quotes from published authors. 